\title{
Close stellar encounters with the Sun from the first Gaia Data Release
}

\author{
Coryn A. L. Bailer-Jones \\ Max Planck Institute for Astronomy, Königstuhl 17, 69117 Heidelberg, Germany \\ email: calj@mpia.de
}

\begin{abstract}
I present preliminary results of searching for close stellar encounters with the Sun using the first Gaia data release. Gl 710 is found to be a much closer encounter than found in preTGAS studies. More detailed results will be reported in an upcoming publication (Bailer-Jones 2017 , in preparation).
\end{abstract}

Keywords. comets:general - Oort cloud - stars: general, kinematics and dynamics - methods: numerical, statistical - surveys: Gaia

\section{Introduction}

Ever since we've been able to measure three-dimensional stellar space velocities, people have wanted to identify stars which have - or which will - come close to the solar system. One motivation is to study the effect of encounters on the Oort cloud, as it is understood that the gravitational perturbations of passing stars is partially responsible for generating the long-period comets. Many searches for close encounters have been published over the past decades, may using Hipparcos (e.g. García-Sánchez et al. 1999, 2001; Dybczyński 2006; Bailer-Jones 2015; Dybczyński \& Berski 2015; Bobylev 2010a,b; Mamajek et al. 2015; Berski \& Dybczyński 2016). Here I report on preliminary results from an on-going study of encounters found using the Gaia data. A more detailed and updated report will appear in a forthcoming publication (Bailer-Jones 2017, in preparation).

\section{Method}

TGAS astrometry (Lindegren et al. 2016) from Gaia Data Release 1 (Gaia Collaboration et al. 2016) was cross-matched with various radial velocity (RV) catalogues which claim measurement precisions better of around $2 \mathrm{~km} \mathrm{~s}^{-1}$ or better (in particular RAVEDR5, Kunder et al. 2017). This yielded a set of about 300000 objects with complete $6 \mathrm{D}$ phase space information (3D position and $3 \mathrm{D}$ velocity). Note that a given star can appear in more than one RV catalogue, so the number of (what I call) objects is larger than the number of (unique) stars. Using the linear motion approximation (LMA; i.e. motion neglecting gravity; see Bailer-Jones 2015), I first computed the time and distance at which each object encounters the Sun (perihelion). 709 objects have perihelia less than $10 \mathrm{pc}$. The distribution of their data and the corresponding standard uncertainties are shown in Figure 1.

I then integrated the orbits of these 709 objects in a Galactic potential in order to compute more accurate perihelia (same model as in Bailer-Jones 2015). By resampling the data for each object and integrating the orbits for all of the resulting surrogates, we properly characterize the (generally asymmetric) distribution in the perihelion distance, time, and speed. I summarize each distribution using the median and the $90 \%$ equaltailed confidence interval (CI). When doing this resampling it is vital to take into account 

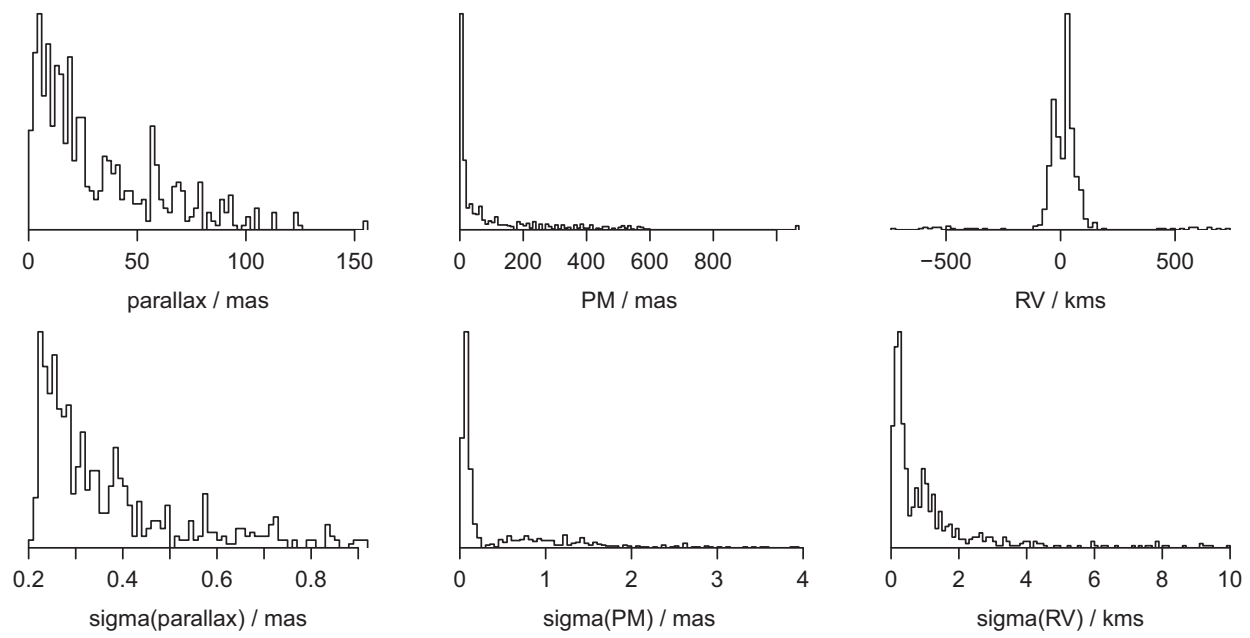

Figure 1. Distribution of the parallax, proper motion, and radial velocity, plus their standard uncertainties, for the 709 objects.
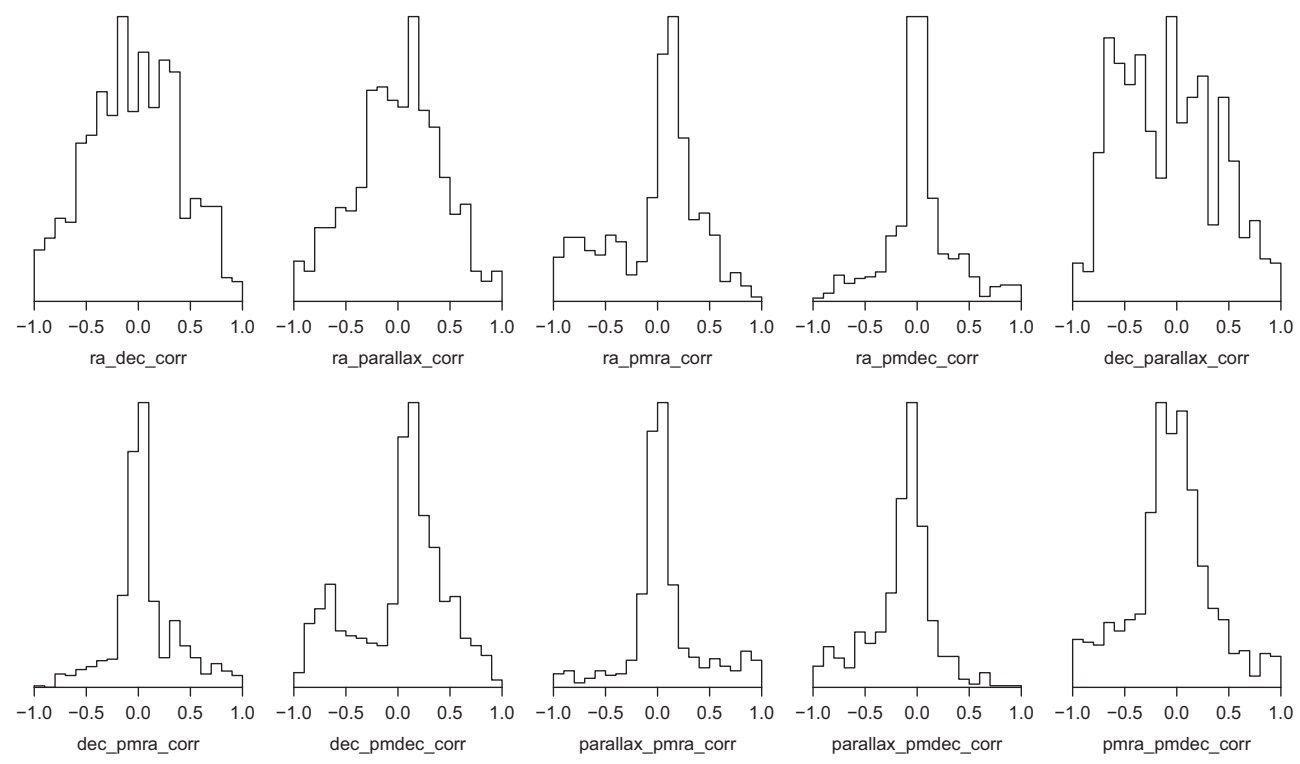

Figure 2. Distribution of the correlations between the astrometric measurements for the 709 objects.

the correlations between the different astrometric parameters for individual objects (as reported in the TGAS catalogue), as these can be very high (see Figure 2).

This procedure does not, of course, find all encounters in my input catalogue which come within $10 \mathrm{pc}$, as the initial LMA-selection it not guaranteed to include all objects which, when properly integrated, would come within $10 \mathrm{pc}$. Yet the LMA turns out to be a good approximation for most stars (the potential deflects paths little over timescales of a few Myr), so this approach is adequate for identifying encounters which come much closer than $10 \mathrm{pc}$. 


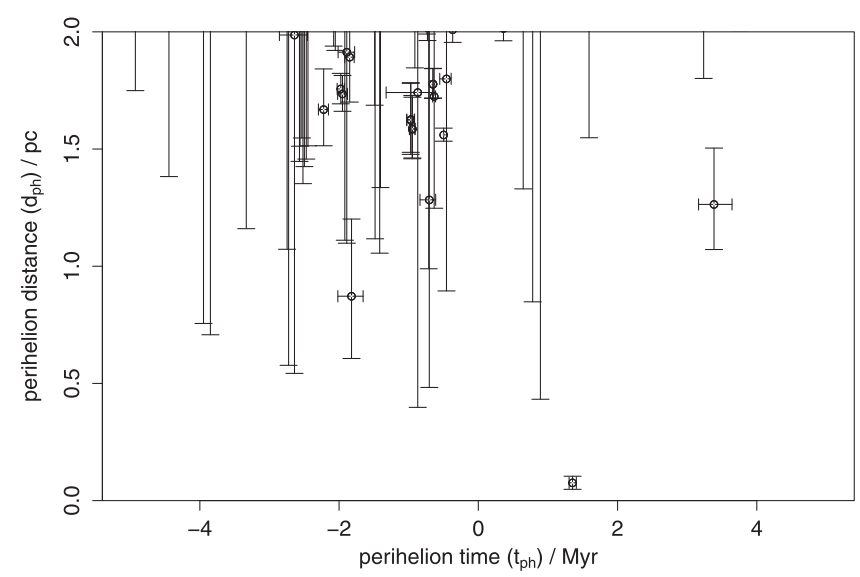

Figure 3. The closest stellar encounters to the Sun found by orbital integration in this study. The open circles show the median of the distribution and the (asymmetric) error bars show the $90 \%$ equal-tailed confidence intervals. This shows objects, not unique stars, so includes some duplicates. The closest encountering star is Gl 710 .
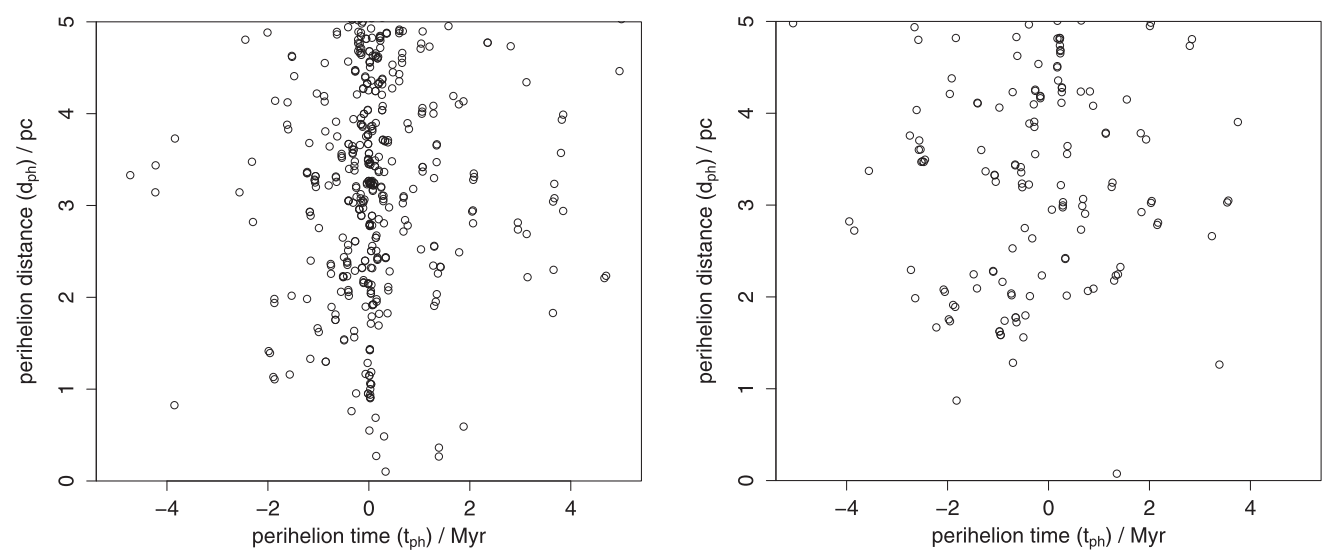

Figure 4. Comparison between object encounters found in this study from TGAS (left) with those found in Bailer-Jones (2015) based on Hipparcos data.

\section{Results}

The perihelion times and distances for the closest encounters found by the orbital integration are shown in Figure 3. This includes some duplicate stars. The closest encounter is Gl 710. This has been known for some time to be a close encounter: most previous studies have found it. Based on Hipparcos- 2 data and a radial velocity of $-13.8 \pm 0.3 \mathrm{~km} \mathrm{~s}^{-1}$, I found a median encounter distance of $0.26 \mathrm{pc}(90 \%$ CI $0.10-0.44 \mathrm{pc})$ (from Bailer-Jones (2015) and its online supplement). TGAS, however, assigns this star a much smaller

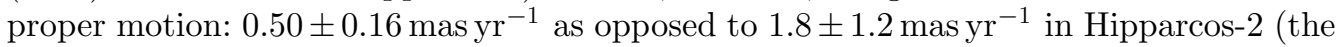
parallax is the same to within $2 \%$ ). Using the same radial velocity, my orbital integration now gives a median perihelion distance of $0.08 \mathrm{pc}(90 \%$ CI $0.05-0.10 \mathrm{pc})$, equivalently $16000 \mathrm{AU}$ (90\% CI 10000-21000 AU).

Berski \& Dybczyński (2016) - who, unsurprisingly, found a very similar perihelion based on the same data and similar method - went on to explore the impact this stars would have on the Oort cloud. Although this close approach would take Gl710 well within the Oort cloud - and its relative velocity at encounter is low $\left(14 \mathrm{~km} \mathrm{~s}^{-1}\right)$ - it is a 
low mass (around $0.6 \mathrm{M}_{\odot}$ ) $\mathrm{K} 7$ dwarf, so its perturbing influence could be less than more distant but more massive encounters.

In my Hipparcos-based study (Bailer-Jones 2015) I found many more close encounters, as can be seen in Figure 4. As discussed in that paper, some of these encounters had dubious astrometric or radial velocity data, and I stressed that one should not blindly use Table 3 as a reliable list. Unfortunately, many of these interesting cases are not in TGAS, primarily because they are too bright (the TGAS bright limit is about $\mathrm{G}=6 \mathrm{mag}$ ) or because there have not yet been enough observations to permit a reliable astrometric solution. This lack of bright stars probably explains why, in the present TGAS study, we see far fewer objects which are currently near perihelion (i.e. with $t_{\mathrm{ph}}$ near to zero in the left panel of Figure 4) compared to the Hipparcos-based study.

It must also be emphasised that the decrease in the number of encounters at larger absolute perihelion times is in part an observational selection effect due to the limiting magnitude of the survey (Hipparcos or TGAS). More distant stars will, in general, have encounters further in the past or present, and these stars are currently fainter and so less likely to be observed. However, some of this decrease in density at large times is, somewhat counter-intuitively, a consequence of the intrinsic spatial and velocity distribution of stars. Such effects are discussed in more detail in the upcoming publication, which also gives an incompleteness-corrected estimate of the current encounter rate.

\section{Conclusions}

A wide-ranging search for close stellar encounters with the solar system can only be done using large, precise, deep astrometric surveys. Gaia is a huge step forward. Although the first data release is limited, it has already allowed us to extend the list of close encounters and improve the precision for known encounters. TGAS is nonetheless limited not only by its depth, but also its complex selection function and bright limit. All of these issues will be addressed by future Gaia data releases. We are, however, already moving into a regime where our studies are limited not by the availability of astrometry, but by the availability of sufficiently accurate radial velocities.

\section{Acknowledgements}

This work has made use of data from the European Space Agency (ESA) mission Gaia (https://www. cosmos.esa.int/gaia), processed by the Gaia Data Processing and Analysis Consortium (DPAC). Funding for the DPAC has been provided by national institutions, in particular the institutions participating in the Gaia Multilateral Agreement. Funding for RAVE (http://www.rave-survey.org) has been provided by institutions of the RAVE participants and by their national funding agencies.

\section{References}

Bailer-Jones C. A. L., Mar. 2015, A\&A, 575, A35

Berski F., Dybczyński P. A., Nov. 2016, A\& A, 595, L10

Bobylev V.V., Mar. 2010a, Astronomy Letters, 36, 220

Bobylev V.V., Nov. 2010b, Astronomy Letters, 36, 816

Dybczyński P. A., Apr. 2006, A\&A, 449, 1233

Dybczyński P. A., Berski F., May 2015, MNRAS, 449, 2459

Gaia Collaboration, Brown A. G. A., Vallenari A., et al., Nov. 2016, A\&\&A, 595, A2

García-Sánchez J., Preston R. A., Jones D. L., et al., Feb. 1999, AJ, 117, 1042

García-Sánchez J., Weissman P. R., Preston R. A., et al., Nov. 2001, A\&AA, 379, 634

Kunder A., Kordopatis G., Steinmetz M., et al., Feb. 2017, AJ, 153, 75

Lindegren L., Lammers U., Bastian U., et al., Nov. 2016, A\& A, 595, A4

Mamajek E. E., Barenfeld S. A., Ivanov V. D., et al., Feb. 2015, ApJ, 800, L17 